\title{
ARTICLE OPEN Biphoton shaping with cascaded entangled-photon sources
}

\author{
Arash Riazi $\mathbb{D}^{1}{ }^{1}$, Changjia Chen ${ }^{1}$, Eric Y. Zhu ${ }^{1}$, Alexey V. Gladyshev $\mathbb{D}^{2}$, Peter G. Kazansky ${ }^{3}$, J. E. Sipe ${ }^{4}$ and Li Qian $^{1}$
}

Quantum entanglement is an integral part of quantum optics and has been exploited in areas such as computation, cryptography and metrology. The entanglement between photons can be present in various degrees of freedom (DOFs), and even the simplest bi-partite systems can occupy a large Hilbert space. Therefore, it is desirable to exploit this multi-dimensional space for various quantum applications by fully controlling the properties of the entangled photons in multiple DOFs. While current entangledphoton sources are capable of generating entanglement in one or more DOFs, there is currently a lack of practical techniques that can shape and control the entanglement properties in multiple DOFs. Here we show that cascading two or more entangled-photon sources with tunable linear media in between allows us to generate photon-pairs whose entanglement properties can be tailored and shaped in the frequency and polarisation domains. We first develop a quantum mechanical model to study the quantum state generated from the cascade structure with special considerations paid to the effects of pump temporal coherence, linear dispersion, and in-structure polarisation transformation applied between the entangled-photon sources. We then experimentally generate photon-pairs with tunable entanglement properties by manipulating the dispersion and birefringence properties of the linear medium placed in between two entangled-photon sources. This is done in an all-fibre, phase stable, and alignment-free configuration. Our results show that the cascade structure offers a great deal of flexibility in tuning the properties of entangled photons in multiple DOFs, opening up a new avenue in engineering quantum light sources.

npj Quantum Information (2019)5:77; https://doi.org/10.1038/s41534-019-0188-1

\section{INTRODUCTION}

Entanglement is an essential resource in quantum optics and can be exploited for quantum information processing ${ }^{1-5}$ and the study of fundamental physics. ${ }^{6-8}$ New developments in quantum optics aim to generate entangled photons whose properties in various degrees of freedom (DOFs) can be tailored and controlled. Frequency and polarisation of photons are robust DOFs often used in practical applications. As a result, a vast number of protocols and platforms have already been developed to exploit these two DOFs; for example, the spectrum of entangled photons (biphotons) has been exploited for scalable quantum information processing ${ }^{1,2,9-11}$ and large alphabet quantum key distribution; ${ }^{12}$ the ability to generate various biphoton polarisation states has also been recognised as a useful resource ${ }^{13-16}$ for tests of local realism ${ }^{6,7}$ and complementarity in physics., 8,17

In light of this, we can envision that the ability to tailor and shape the entanglement properties of biphotons in both frequency and polarisation DOFs would allow us to increase the amount of information that can be encoded into a biphoton state, ${ }^{5}$ enabling a variety of new applications in quantum optics. In order to achieve this goal, we first need spectral and polarisation shaping techniques for biphotons that are compatible with each other; these techniques should also be implementable in an integrated and scalable fashion.

So far, various techniques of biphoton spectral shaping have been demonstrated with spatial light modulators, ${ }^{18,19}$ spectral filtering, ${ }^{20-22}$ and tailoring the phase-matching structure of the nonlinear medium itself. ${ }^{23,24}$ However, these techniques either introduce undesirable loss due to coupling and filtering, ${ }^{20-22}$ or impose considerable complications in the precise fabrication of the nonlinear structure. ${ }^{23,24}$ Additionally, some of these techniques $^{18,19}$ cannot yet be integrated with waveguide-based biphoton sources, and therefore cannot take advantage of the greater mode confinement.

Various techniques to shape the polarization state of biphotons have also been demonstrated, typically through a combination of biphoton interference ${ }^{14-16}$ unitary polarisation transformation, ${ }^{14,15}$ decoherence, ${ }^{14,16}$ and spatial mode selection. ${ }^{16}$ However, these techniques have all been implemented using free-space setups and cannot be integrated with waveguide-based biphoton sources in a single platform. Moreover, precise beam alignment, spatial filtering, and phase stabilization are required for these techniques, which make them difficult to implement in integrated photonics. Finally, these techniques have not been shown to be simultaneously compatible with spectral tailoring. In fact, no practical approach to shape the biphotons simultaneously in both the spectral and polarization domains has been demonstrated.

In this paper, we demonstrate a technique that can shape biphoton states in both the frequency and polarisation domains by cascading two fibre-based entangled-photon sources ${ }^{25,26}$ with a linear medium placed in between, which we refer to as the middle section (see Fig. 1a). Our cascade structure, which is essentially a nonlinear interferometer, ${ }^{27}$ can be pumped either with a long- or short-coherence-time laser, with each option providing a specific functionality for shaping the properties of biphotons. The spectrum and polarisation state of the biphotons generated from the cascade structure can be tailored by altering the dispersion and birefringence of the linear middle section. The

\footnotetext{
${ }^{1}$ Department of Electrical and Computer Engineering, University of Toronto, 10 King's College Rd., Toronto M5S 3G4, Canada; ${ }^{2}$ Fibre Optics Research Center, Russian Academy of Sciences, 38 Vavilov Street, 119333 Moscow, Russia; ${ }^{3}$ Optoelectronics Research Centre, University of Southampton, Southampton SO17 1 BJ, UK and ${ }^{4}$ Department of Physics, University of Toronto, 60 St. George St., Toronto M5S 1A7, Canada

Correspondence: Arash Riazi (arash.riazi@mail.utoronto.ca)
}

Received: 18 March 2019 Accepted: 12 August 2019

Published online: 10 September 2019 

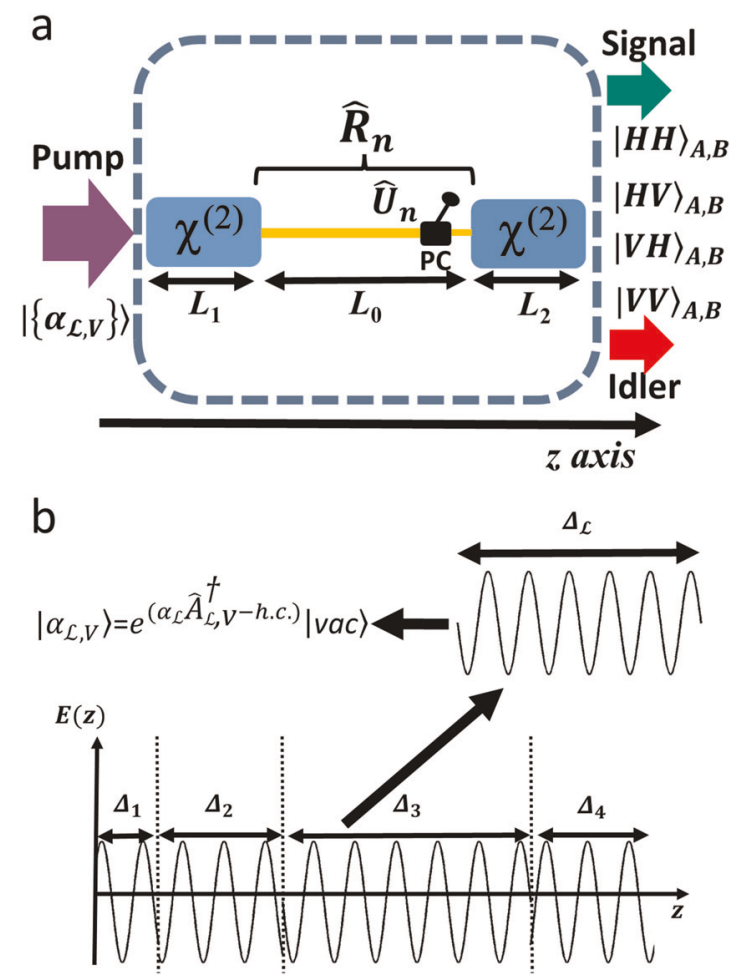

Fig. 1 a A two-segment cascade structure made up of two second-order nonlinear media of the lengths $L_{1}$ and $L_{2}$; the pump coherent packets with $V$ polarisation, $\left|\left\{a_{\mathcal{L}, V}\right\}\right\rangle$, enter the structure at $z=0$. Depending on the transformation in the middle section $\hat{R}_{n}$, the polarisation states of the downconverted photon pairs at the output of the cascade structure could be a superposition of all four states $\left(|\mathrm{HH}\rangle_{\omega_{A}, \omega_{B}},|\mathrm{HV}\rangle_{\omega_{A}, \omega_{B}},|\mathrm{VH}\rangle_{\omega_{A}, \omega_{B}},|\mathrm{VV}\rangle_{\omega_{A}, \omega_{B}}\right)$. b The quasimonochromatic model of the pump field ${ }^{49}$; the pump consists of coherent packets, each of which has a length of $\Delta_{\mathcal{L}}$. The initial phase of the electric field within each packet changes randomly from packet to packet. ${ }^{50}$

all-fibre common-path configuration used here eliminates major issues in biphoton shaping, such as the requirement for beam alignment, coupling and filtering loss, and phase stabilization. More importantly, spectral and polarisation shaping techniques are now compatible with each other and can be simultaneously implemented in such a structure.

It is worth mentioning that the cascade structure we use here belongs to a more general class known as $\mathrm{SU}(1,1)$ nonlinear interferometer. ${ }^{27,28}$ The high-gain regime of these interferometers has been extensively studied ${ }^{27-31}$ and utilised ${ }^{32-34}$ to obtain the Heisenberg limit in phase measurement. On the other hand, the spontaneous regime of these interferometers has also been studied both theoretically, ${ }^{28,35-37}$ and experimentally ${ }^{38-41}$ to investigate more abstract concepts such as "induced coherence" effect. $^{38,42,43}$ These studies have since found their applications in measuring absorption, ${ }^{44}$ refractive index, ${ }^{45}$ and dispersion ${ }^{46}$ of linear media.

Our work, however, differs from previous studies in that we utilise cascaded biphoton sources (a nonlinear interferometer) in two DOFs (frequency and polarisation) to generate biphotons with tunable entanglement properties in spectral and polarisation domains. Furthermore, the quantum mechanical treatment of the cascade structure we present here is more comprehensive as it takes into account the collective effects of the pump temporal coherence, the chromatic dispersion in the structure, and the polarisation transformations on the biphoton state generated from the cascade structure. Finally, our formulation can be generalised to other waveguide-based entangled-photon sources, including those in integrated photonics.

The organization of the paper is as follows: We first present a quantum mechanical model for the biphoton state at the output of the cascade structure, taking into account (1) the temporal coherence of the pump (referred to below simply as the pump coherence), (2) the dispersion properties of both linear and nonlinear segments, and (3) the polarisation transformation applied in the linear middle section of the cascaded structure. We then use our model to study the spectrum and polarisation state of the biphotons under various pump coherence conditions and polarisation transformations caused by the middle (linear) section. Finally, using two periodically-poled silica fibres ${ }^{26}$ (PPSFs) as biphoton sources, we experimentally demonstrate: (1) the ability to generate biphotons with modified spectra, for various pump coherence conditions and the linear properties of the middle section; and (2) the ability to generate various biphoton polarisation states with properties such as tunable degree of polarisation entanglement.

\section{RESULTS}

The cascade structure and theoretical framework

The general two-segment cascade structure is shown in Fig. 1a. Two identical second-order nonlinear segments are connected via a "middle section" consisting of a linear optical medium, and an inline polarisation controller (PC). By using the nonlinear waveguide scattering theory presented earlier, ${ }^{47,48}$ we model biphoton generation in our cascade structure. For our formulation, we consider only type-II SPDC phase-matching; however, it can be trivially generalised to other SPDC phase-matchings as well. We define horizontal $(H)$, and vertical $(V$ ) polarisations according to the principal axes (polarisation eigenmodes) of the nonlinear segments. Note that due to the polarisation transformation in the middle section, light polarised along one of the principal axes in the first nonlinear segment will not generally be polarised along the same principal axes of the second nonlinear segment.

We model the pump in Fig. 1a as a quasi-monochromatic field with a finite coherence time of $\tau_{c}$. In our model, the pump field is a succession of coherent packets ${ }^{49,50}$ (see Fig. 1b) in which the electric field oscillates with a constant angular frequency of $\bar{\omega}_{p}$ (see Supplement, Section 2); the initial phase of the electric field within each packet is assumed to be constant, however, it is statistically distributed for each packet. ${ }^{49,50}$ The generalised creation operator for the $\mathcal{L}^{\text {th }}$ pump packet with polarisation $S$ is denoted by $\hat{A}_{\mathcal{L}, S}^{\dagger}=\int \mathrm{d} k_{P} f_{\mathcal{L}}^{*}\left(k_{P}\right) \hat{a}_{P S k_{p}}^{\dagger}$, where $f_{\mathcal{L}}\left(k_{P}\right)$ includes the spectral behaviour of that packet (see Supplement, Section 2) and is normalised according to $\int d k_{P}\left|f_{\mathcal{L}}\left(k_{P}\right)\right|^{2}=1$. The quantum state of each pump packet incident on the structure is taken to be a coherent state in vertical polarisation and can be written as $\left|a_{\mathcal{L}, V}\right\rangle=e^{a_{\mathcal{L}} \hat{A}_{\mathcal{L}, V}^{\dagger}-h_{\text {.c. }}}|\mathrm{vac}\rangle$, where $\left|a_{\mathcal{L}}\right|^{2}$ is the average photon number inside the $\mathcal{L}^{\text {th }}$ pump packet. Since the field operators of different packets commute due to $\left[\hat{A}_{\mathcal{L}, \mathrm{v}}, \hat{A}_{\mathcal{L}^{\prime}, \mathrm{v}}^{\dagger}\right]=\delta_{\mathcal{L}, \mathcal{L}^{\prime}}$ (see Supplement, Section 2), we can write down the quantum state of the pump at the input of the cascade structure as:

$\left|\left\{a_{\mathcal{L}, V}\right\}\right\rangle=e^{\sum_{\mathcal{L}}\left(a_{\mathcal{L}} \hat{A}_{\mathcal{L}, V}^{\dagger}-\text { h.c. }\right)} \mid$ vac $\rangle$

In the weak conversion limit with negligible probability of multipair generation, the quantum state of the downconverted light for an individual nonlinear segment with a length of $L_{1}$ can be 
described as:

$$
\begin{array}{r}
\left.\left|\Psi_{\text {seg }}\right\rangle=\mid \text { vac }\right\rangle_{\omega_{A}, \omega_{B}}+\sum_{\mathcal{L}} a_{\mathcal{L}} \int \mathrm{d} \omega_{P} \mathrm{~d} \omega_{A} \mathrm{~d} \omega_{B} \mathcal{A}_{0}^{\text {type II }}\left(\omega_{P}, \omega_{A}, \omega_{B}\right) f_{\mathcal{L}}\left(\omega_{P}\right) \delta\left(\omega_{P}-\omega_{A}-\omega_{B}\right) \\
\times\left[L_{1} \operatorname{sinc}\left(\frac{\Delta k_{\mathrm{vVV}}^{(1)} L_{1}}{2}\right) \hat{a}_{A H}^{\dagger} \hat{a}_{B V}^{\dagger}+e^{i \Lambda\left(\omega_{A}, \omega_{B}\right)} L_{1} \operatorname{sinc}\left(\frac{\Delta k_{\mathrm{vH}}^{(1)} L_{1}}{2}\right) \hat{a}_{A V}^{\dagger} \hat{a}_{B H}^{\dagger}\right]|\mathrm{vac}\rangle_{\omega_{A}, \omega_{B}},
\end{array}
$$

where the sum is over all the pump packets, and $\omega_{P}, \omega_{A}$, and $\omega_{B}$ are the angular frequencies of the pump, signal, and idler fields, respectively; $\hat{a}_{A S^{\prime}}^{\dagger}\left(\hat{a}_{B S^{\prime \prime}}^{\dagger}\right)$ is the creation operator of the signal (idler) mode with $S^{\prime}\left(S^{\prime \prime}\right)$ polarisation; the quantity $\mathcal{A}_{0}\left(\omega_{P}, \omega_{A}, \omega_{B}\right)$ includes the nonlinear susceptibilities and other phase factors (see Supplement, Section 5); $\Delta k_{S S^{\prime} S^{\prime \prime}}^{(m)}=k_{P S}^{(m)}\left(\omega_{P}\right)-k_{A S^{\prime}}^{(m)}\left(\omega_{A}\right)-k_{B S^{\prime \prime}}^{(m)}\left(\omega_{B}\right)-k_{\mathrm{QPM}}$, where the first and the second subscripts of the wavevector refer to the field and its polarisation, respectively, while the superscript $m$ refers to the nonlinear media; $k_{\mathrm{QPM}}$ is the quasi-phase-matching wavevector of the nonlinear medium. Note that $\Delta k_{\mathrm{VHV}}^{(1)} L_{1}$ [Eq. (2)] in general is different from $\Delta k_{\mathrm{WH}}^{(1)} L_{1}$; however, due to the slowlyvarying nature of the sinc function with respect to frequency [in comparison with other phase factors in Eq. (2)] and small difference between $\Delta k_{\mathrm{VHV}}^{(1)} L_{1}$ and $\Delta k_{\mathrm{VVH}}^{(1)} L_{1}$ over the phase-matching (full width at half maximum) bandwidth of the signal and idler, we have $\operatorname{sinc}\left(\frac{\Delta k_{\mathrm{VHV}}^{(1)} L_{1}}{2}\right) \approx \operatorname{sinc}\left(\frac{\Delta k_{\mathrm{WH}}^{(1)} L_{1}}{2}\right)$. Finally, the quantity $\Lambda\left(\omega_{A}, \omega_{B}\right)$ [related to group birefringence, see ref. ${ }^{51}$ ] is defined as:

$\Lambda\left(\omega_{A}, \omega_{B}\right)=\left[k_{A V}^{(1)}\left(\omega_{A}\right)-k_{A H}^{(1)}\left(\omega_{A}\right)+k_{B H}^{(1)}\left(\omega_{B}\right)-k_{B V}^{(1)}\left(\omega_{B}\right)\right] \frac{L_{1}}{2}$.

Henceforth, we shall drop the angular frequency notation for the wavevector $k(\omega)$; for $\mathcal{A}_{0}^{\text {type Il }}\left(\omega_{P}, \omega_{A}, \omega_{B}\right)$ and $\delta\left(\omega_{P}-\omega_{A}-\omega_{B}\right)$, we simply write $\mathcal{A}_{0}^{\text {typell, }}$ and $\delta$, respectively.

Note that the SPDC emission within the PPSF [see Eq. (2)] results in both signal and idler photons travelling in one spatial mode. However, we can distinguish the two based on their angular frequencies; photons with angular frequencies greater than $\frac{\overline{\omega_{p}}}{2}$ are called signal; otherwise, they are called idler. Note that our definition of signal and idler best describes cases with narrowband pump [e.g. continuous wave(cw) pump].

We also remark that for most second-order nonlinear media, the biphoton state generated from type-II SPDC is not polarisationentangled due to the walk-off caused by the frequencydependent factor $e^{\mathrm{i} \wedge\left(\omega_{A}, \omega_{B}\right)}$ in Eq. (2) [see ref. ${ }^{51}$ ]; however, because of the unique dispersive properties of poled-fibre $\left[\Lambda\left(\omega_{A}, \omega_{B}\right) \ll 1\right.$, see ref. $\left.{ }^{51}\right]$, type-II SPDC in PPSFs allows for the direct generation of polarisation-entangled photon-pairs. ${ }^{26,51,52}$ Since we are using PSSF as our nonlinear medium, whenever type-II SPDC is involved, the biphoton state is polarisation-entangled.

The state of the generated biphotons in the cascade structure Now we consider a cascade of two identical nonlinear segments pumped for type-II SPDC; the two nonlinear segments are connected via a linear medium (with a length of $L_{0}$ ), by which we shape the spectrum and polarisation state of the biphotons generated from the cascade structure. We derive the quantum state of the biphotons by employing several assumptions: (1) The collective transformation of the middle section in Jones space can be modelled by two consecutive transformations: A phase accumulation $e^{i k_{n}^{(0)} L_{0}} \hat{l}$, where $\hat{l}$ is a $2 \times 2$ identity matrix, and a unitary polarisation transformation $\hat{U}_{n}=\left(\begin{array}{ll}U_{1 n} & U_{2 n} \\ U_{3 n} & U_{4 n}\end{array}\right)$ [see ref. ${ }^{53}$ ], where the subscripts $n=P, A$, and $B$. Accordingly, the collective transformation of the middle section (see Fig. 1a) becomes: $\hat{R}_{n}=$ $e^{i k_{n}^{(0)} L_{0}}\left(\begin{array}{ll}U_{1 n} & U_{2 n} \\ U_{3 n} & U_{4 n}\end{array}\right)$ (see Supplement, Section 3); (2) The middle section is assumed to have a weak wavelength-dependent birefringence such that $\hat{R}_{A}=\hat{R}_{B} \neq \hat{R}_{p}$. In other words, the signal and idler are assumed to undergo the same polarisation transformation, while the pump does not necessarily do so; (3) While the presence of the middle section may result in the pump polarisation having both $H$ and $V$ components when entering the second nonlinear segment, due to the phase-matching constraint (wavelength) for type-II SPDC, the $H$ component of the pump will not contribute to other SPDC types (such as type-0) in the second segment (see Supplement, Section 5.1). So the effect of the middle section is to merely transform the polarisation state of the biphotons that could be generated in the first segment.

Under these assumptions, the quantum state of the biphotons at the output of the cascade structure can be written as a linear superposition of all possible biphoton states,

$$
\begin{aligned}
\left|\psi_{\mathrm{CAS}}\right\rangle=|\mathrm{vac}\rangle_{\omega_{A}, \omega_{B}}+\sum_{\mathcal{L}}\left\{\int \mathrm{d} \omega_{A} \mathrm{~d} \omega_{B} \phi_{\mathcal{L}, H H}\left(\omega_{A}, \omega_{B}\right) \hat{a}_{\mathrm{AH}}^{\dagger} \hat{a}_{\mathrm{BH}}^{\dagger}\right. \\
\quad+\int \mathrm{d} \omega_{A} \mathrm{~d} \omega_{B} \phi_{\mathcal{L}, \mathrm{HV}}\left(\omega_{A}, \omega_{B}\right) \hat{a}_{\mathrm{AH}}^{\dagger} \hat{a}_{\mathrm{BV}}^{\dagger}+\int \mathrm{d} \omega_{A} \mathrm{~d} \omega_{B} \phi_{\mathcal{L}, \mathrm{VH}}\left(\omega_{A}, \omega_{B}\right) \hat{a}_{\mathrm{AV}}^{\dagger} \hat{a}_{\mathrm{BH}}^{\dagger} \\
\left.\quad+\int \mathrm{d} \omega_{A} \mathrm{~d} \omega_{B} \phi_{\mathcal{L}, W}\left(\omega_{A}, \omega_{B}\right) \hat{a}_{\mathrm{AV}}^{\dagger} \hat{a}_{\mathrm{BV}}^{\dagger}\right\}|\mathrm{vac}\rangle_{\omega_{A}, \omega_{B}},
\end{aligned}
$$

where $\phi_{\mathcal{L} \text {.S' }{ }^{\prime} \mathcal{S}^{\prime \prime}}\left(\omega_{A}, \omega_{B}\right)$ is the biphoton wavefunction, corresponding to the $\mathcal{L}^{\text {th }}$ pump packet, which can be determined by the Hamiltonian treatment of the cascade structure (see Supplement, Section 4-6). Henceforth neglecting the vacuum contribution, we can write the quantum state of the biphotons generated from the cascade structure as:

$$
\begin{aligned}
& \left|\psi_{\text {CAS }}\right\rangle=\sum_{\mathcal{L}} a_{\mathcal{L}} \int \mathrm{d} \omega_{P} \mathrm{~d} \omega_{A} \mathrm{~d} \omega_{B} \mathcal{A}_{0}^{\text {type II }} f_{\mathcal{L}}\left(\omega_{P}\right) L_{1} \operatorname{sinc}\left(\frac{\Delta k_{\text {VVV }}^{(1)} L_{1}}{2}\right) \delta \\
& \times\left\{-e^{\mathrm{i} \Gamma_{i}}\left[\left(U_{4 A} U_{3 B}\right)^{*}+e^{\mathrm{i} \wedge}\left(U_{4 B} U_{3 A}\right)^{*}\right]|\mathrm{HH}\rangle_{\omega_{A}, \omega_{B}}\right. \\
& +\left[\left(U_{4 A} U_{1 B}\right)^{*}+e^{\mathrm{i} \Lambda}\left(U_{3 A} U_{2 B}\right)^{*}+U_{4 p} e^{\mathrm{i}\left(\Delta k^{(0)} L_{0}+\Delta k_{\mathrm{VHV}}^{(1)} L_{1}\right)}\right]|\mathrm{HV}\rangle_{\omega_{A}, \omega_{B}} \\
& +e^{2 \mathrm{i} \Lambda}\left[\left(U_{2 A} U_{3 B}\right)^{*}+e^{\mathrm{i} \Lambda}\left(U_{1 A} U_{4 B}\right)^{*}+e^{-i \Lambda} U_{4 p} e^{i\left(\Delta k^{(0)} L_{0}+\Delta k_{\mathrm{VHV}}^{(1)} L_{1}\right)}\right]|\mathrm{VH}\rangle_{\omega_{A}, \omega_{B}} \\
& \left.-e^{\mathrm{i} \Gamma_{s}}\left[\left(U_{2 A} U_{1 B}\right)^{*}+e^{\mathrm{i} \wedge}\left(U_{2 B} U_{1 A}\right)^{*}\right]|\mathrm{V}\rangle \omega_{A}, \omega_{B}\right\} \text {, }
\end{aligned}
$$

where we assumed the two nonlinear media have the same length $\left(L_{1}=L_{2}\right)$ and identical dispersion properties [i.e. $\left.k_{H(V)}^{(1)}(\omega)=k_{H(V)}^{(2)}(\omega)\right]$; here $\Gamma_{A(B)}=\left(k_{A(B) V}^{(1)}-k_{A(B) H}^{(1)}\right) L_{1}$, and $\Delta k^{(0)} L_{0}=$ $\left(k_{P}^{(0)}-k_{A}^{(0)}-k_{B}^{(0)}\right) L_{0}$ is the phase introduced by the middle section. Note that due to the polarisation transformation in the middle section, $\phi_{\mathcal{L}, H H}\left(\omega_{A}, \omega_{B}\right)$ and $\phi_{\mathcal{L}, W}\left(\omega_{A}, \omega_{B}\right)$ are now nonzero and the extra biphoton polarisation states $|\mathrm{HH}\rangle_{\omega_{A}, \omega_{B}}$ and $|\mathrm{VV}\rangle_{\omega_{A}, \omega_{B}}$ appear at the output of the cascade structure. Moreover, $\phi_{\mathcal{L}, H V}\left(\omega_{A}, \omega_{B}\right)$ and $\phi_{\mathcal{L}, V H}\left(\omega_{A}, \omega_{B}\right)$ [the prefactors of $|\mathrm{HV}\rangle_{\omega_{A}, \omega_{B}}$ and $|V H\rangle_{\omega_{A}, \omega_{B}}$ in Eq. (5)] now contain contributions from both nonlinear segments, which eventually leads to interference between the biphoton amplitudes from the two nonlinear segments.

\section{Biphoton spectrum}

In this section, we assume there is no polarisation transformation in the middle section [i.e. $\hat{R}_{n}=e^{i k_{n}^{(0)} L_{0}}\left(\begin{array}{ll}1 & 0 \\ 0 & 1\end{array}\right)$ with $n=A, B$, and only focus on the spectrum of the biphotons generated from the cascade structure in that limit. For this case, if we assume the nonlinear media have $\Lambda\left(\omega_{A}, \omega_{B}\right) \ll 1$ [see Eq. (6)], the quantum state of the biphotons generated from the cascade structure 
4

becomes:

$$
\begin{aligned}
\left|\psi_{\text {CAS }}\right\rangle=\sum_{\mathcal{L}} & a_{\mathcal{L}} \int \mathrm{d} \omega_{P} \mathrm{~d} \omega_{A} \mathrm{~d} \omega_{B} \mathcal{A}_{0}^{\text {type II }} f_{\mathcal{L}}\left(\omega_{P}\right) L_{1} \operatorname{sinc}\left(\frac{\Delta k_{\mathrm{VHV}}^{(1)} L_{1}}{2}\right) \delta \\
& \times\left(1+U_{4 P} e^{\mathrm{i}\left(\Delta k^{(0)} L_{0}+\Delta k_{\mathrm{VHV}}^{(1)} L_{1}\right)}\right)\left\{|\mathrm{HV}\rangle_{\omega_{A}, \omega_{B}}+|\mathrm{VH}\rangle_{\omega_{A}, \omega_{B}}\right\} .
\end{aligned}
$$

Here $U_{4 P}$ is the fourth element of the transformation matrix $\hat{U}_{P}$, which we write as $\left|U_{4 P}\right| e^{i \phi_{P}}$. We now study the relative emission spectrum of the biphotons by expanding the total biphoton brightness $B_{\text {tot }}=\left\langle\psi_{\text {CAS }} \mid \psi_{\text {CAS }}\right\rangle$. As the expression for $B_{\text {tot }}$ involves the statistical phases of different pump packets, we first average $B_{\text {tot }}$ over the ensemble of sequences of the pump packets according to

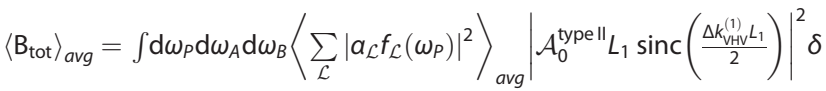

$$
\begin{aligned}
& \times\left|1+U_{4 P} e^{\mathrm{i}\left(\Delta k^{(0)} L_{0}+\Delta k_{\mathrm{VHV}}^{(1)} L_{1}\right)}\right|^{2}
\end{aligned}
$$

We next replace the ensemble average $\left\langle\sum_{\mathcal{L}}\left|a_{\mathcal{L}} f_{\mathcal{L}}\left(\omega_{P}\right)\right|^{2}\right\rangle_{\text {avg }}$ with $|a|^{2}\left|f\left(\omega_{P}\right)\right|^{2}$, where $|a|^{2}$ is the number of photons in the entire pump packets (see Supplement, Section 8); $\left|f\left(\omega_{P}\right)\right|^{2}$ is now the spectral lineshape of the pump, which is assumed to be Lorentzian. ${ }^{49}$ Note that the integral of the form $\int \mathrm{d} \omega_{P} e^{i\left(\Delta k^{(0)} L_{0}+\Delta k_{v H V}^{(1)} L_{1}\right)}\left|f\left(\omega_{P}\right)\right|^{2}$ that appears in Eq. (7) is related to the first-order coherence of the pump, $g^{(1)}(\tau)$ [see ref. ${ }^{49}$ ]. Given that, we can re-write Eq. (7) as:

$$
\begin{aligned}
\left\langle\mathrm{B}_{\text {tot }}\right\rangle_{\text {avg }}=\int & \mathrm{d} \omega_{P} \mathrm{~d} \omega_{A} \mathrm{~d} \omega_{B}\left|\mathcal{A}_{0}^{\text {type } \|} \operatorname{af}\left(\omega_{P}\right) L_{1} \operatorname{sinc}\left(\frac{\Delta k_{\mathrm{VHV}}^{(1)} L_{1}}{2}\right)\right|^{2} \delta \\
& \times\left\{1+\left|U_{4 P}\right|^{2}+2\left|U_{4 P}\right| e^{\frac{-\left|\Delta \Delta_{0}+\Delta \tau_{1}\right|}{{ }^{T_{C}}}} \cos \left(\Delta k^{(0)} L_{0}+\Delta k_{\mathrm{VHV}}^{(1)} L_{1}+\Phi_{P}\right)\right\},
\end{aligned}
$$

where the factor $e^{\frac{-\left|\Delta \tau_{0}+\Delta \tau_{1}\right|}{\tau_{C}}}$ appears in Eq. (8) as a result of first-order coherence function of a pump field with coherence time of $\tau_{c}$ (see Supplement, Section 8); $\Delta \tau_{0}\left(\Delta \tau_{1}\right)$ is the group delay difference between pump and biphotons in the middle section (first nonlinear medium), which can be expressed as:

$\Delta \tau_{0 / 1}=\tau_{0 / 1, P}-\frac{1}{2}\left(\tau_{0 / 1, A}+\tau_{0 / 1, B}\right)=\left(\left.\frac{\mathrm{d} k^{(0 / 1)}}{\mathrm{d} \omega}\right|_{\bar{\omega}_{p}}-\left.\frac{\mathrm{d} k^{(0 / 1)}}{\mathrm{d} \omega}\right|_{\frac{\bar{\omega}_{p}}{2}}\right) L_{0 / 1}$,

where $\left.\frac{d k^{(0 / 1)}}{d \omega}\right|_{\omega^{\prime}}$ is the first-order dispersion of the middle section/ PPSF at frequency $\omega^{\prime}$. The factor $e^{\frac{-\left|\Delta \tau_{0}+\Delta \tau_{1}\right|}{\tau_{C}}}$ in Eq. (8) determines to what extent the biphoton amplitudes from two nonlinear segments interfere. Note that the integrand in Eq. (8) corresponds to the biphoton spectrum (or joint spectral intensity). In the following subsections, we study the biphoton spectrum under two different pump coherence conditions.

Biphoton spectrum: coherent cascading. When $\left|\Delta \tau_{0}+\Delta \tau_{1}\right| \leq \tau_{C}$, the pump field remains coherent throughout both nonlinear segments; we call this mode of operation "coherent cascading". Here the biphoton amplitudes from the two different nonlinear segments interfere with each other, resulting in fringes in the biphoton spectrum (see Supplement, Section 8). Adding more nonlinear segments (Fig. 2a) results in more interference terms, which gives us greater flexibility in shaping the biphoton spectrum. As an example, we could generate biphotons with discrete frequency modes (in the form of a frequency comb ${ }^{20,22}$ ) by cascading three PPSFs whose spectra are initially continuous (Fig. 2b, c). Note that the spacing between the frequency modes in Fig. $2 c$ can be controlled by tailoring the dispersion of the middle section, without resorting to any spectral filtering or modification of the nonlinear media. It is also worth mentioning that since we are utilising type-Il SPDC and using PPSFs as our nonlinear media $\left[\Lambda\left(\omega_{A}, \omega_{B}\right) \ll 1\right.$, Eq. (3)], biphotons generated from the cascade structure (Fig. 2c) are also entangled in the polarisation DOF as well. ${ }^{26,51}$

When $\left|\Delta \tau_{1}\right| \leq \tau_{C}$ and the middle section has no dispersion (equivalent to $L_{0}=0$ ), the coherent cascade of two nonlinear segments becomes equivalent to a longer biphoton source with the total nonlinear interaction length of $L_{\mathrm{NL}}=L_{1}+L_{2}=2 L_{1}$. In this case, the brightness of the biphotons generated in the cascade structure increases by a factor of $2^{3 / 2}$ (scaling with $L_{\mathrm{NL}}^{3 / 2}$, see ref. ${ }^{48}$ ) with respect to the individual nonlinear segment, while the emission bandwidth [now determined by $\operatorname{sinc}^{2}\left(\Delta k_{\mathrm{VHV}}^{(1)} L_{1}\right)$ ] is reduced by a factor of $2^{1 / 2}$ (scaling with $L_{N L}^{-1 / 2}$, see ref. ${ }^{48}$ ) with respect to each of individual nonlinear segment. Note that both of the scaling factors mentioned here generally applies for degenerate SPDC processes in which the signal and idler have the same polarisation. However, as the group birefringence of PPSF is negligible ${ }^{51}$ over the bandwidth of the downconverted photons (see Supplement, Section 8.2), the scaling factors mentioned above also apply for the type-II SPDC phase-matching in the case of PPSF. Figure 3 shows the brightness ( $3 a$ ) and the emission bandwidth (3b) of $N$ identical PPSFs that are coherently cascaded. As can be seen in Fig. 3b, coherent cascade of multiple nonlinear segments (equivalent to increasing the length of the nonlinear medium) reduces the emission bandwidth of the biphotons, which is particularly undesirable for broadband biphoton sources; however, we will show in the following that this issue can be overcome through incoherent cascade of multiple nonlinear segments.

Biphoton spectrum: incoherent cascading. When $\left|\Delta \tau_{1}\right| \leq \tau_{C} \ll\left|\Delta \tau_{0}\right|$, which we refer to as "incoherent cascading", the factor $e^{\frac{-\left|\Delta \tau_{0}+\Delta \tau_{1}\right|}{\tau_{C}}}$ in Eq. (8) vanishes and the biphoton brightness simplifies to:

$$
\left\langle B_{\text {tot }}\right\rangle_{\text {avg }}=\int \mathrm{d} \omega_{P} \mathrm{~d} \omega_{A} \mathrm{~d} \omega_{B}\left|\mathcal{A}_{0}^{\text {type II }} \operatorname{af}\left(\omega_{P}\right) L_{1} \operatorname{sinc}\left(\frac{\Delta k_{\mathrm{VHV}}^{(1)} L_{1}}{2}\right)\right|^{2} \delta \times\left\{1+\left|U_{4 P}\right|^{2}\right\} .
$$

With such a low coherence pump, the biphoton amplitudes from the two nonlinear segments will not interfere, and the brightness of the biphotons becomes twice that of an individual nonlinear segment as $\left|U_{4 P}\right| \rightarrow 1$. More generally, the brightness increases linearly with respect to the total nonlinear interaction length in the cascade structure. On the other hand, the emission bandwidth of the biphotons [determined by $\operatorname{sinc}^{2}\left(\frac{\Delta k_{\mathrm{VVV}}^{(1)} L_{1}}{2}\right)$ ] remains the same as the emission bandwidth of an individual segment. As the number of identical cascaded nonlinear segments $N$ increases, the emission bandwidth remains constant, while the brightness increases linearly (Fig. 3). This suggests that with incoherent cascading, we can arbitrarily increase the brightness of the biphoton source without sacrificing the emission bandwidth of the biphotons which is in contrast to coherent cascading, where increasing the total nonlinear interaction length $L_{\mathrm{NL}}$ was accompanied by a reduction in the emission bandwidth.

\section{Biphoton polarisation state}

In the following subsections, we study the effect of cascading on the degree of polarisation entanglement (quantified by concurrence $^{54}$ ) and the polarisation state of the biphotons generated from the cascade structure. Although our approach can be applied to all SPDC phase-matching processes, in the interest of brevity, we discuss only the cases where type-II SPDC process occurs in both nonlinear media. As in previous cases, we account for the 


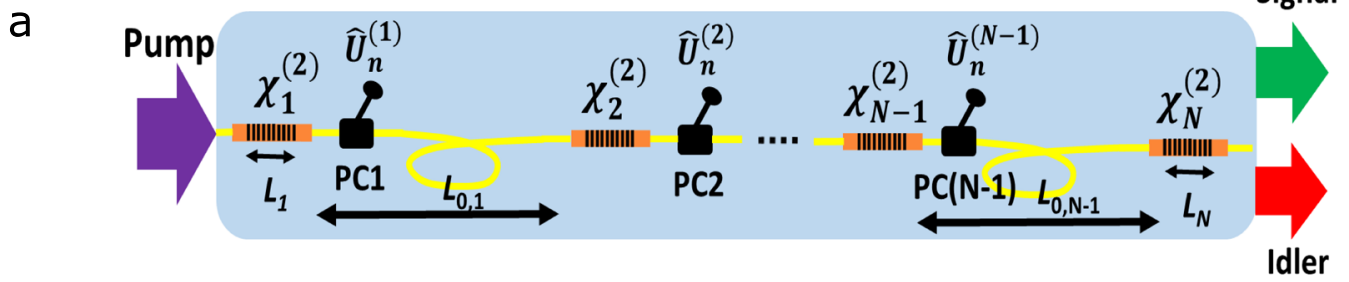

b

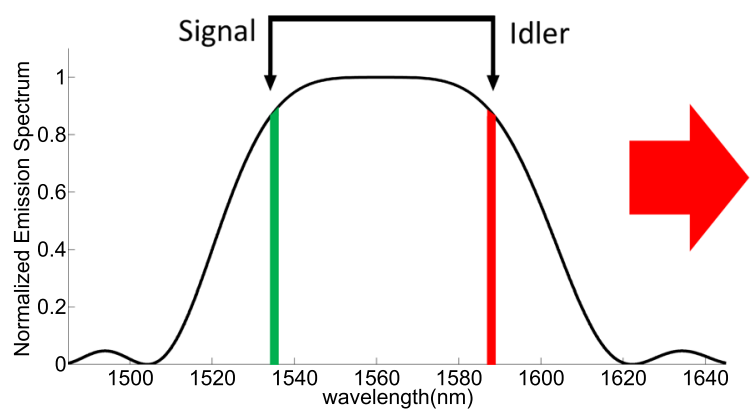

C

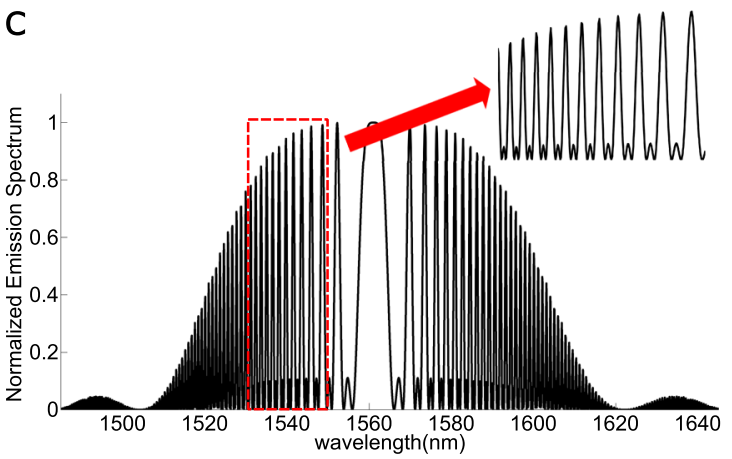

Fig. 2 a A generalised cascade structure consisting of $N$ nonlinear segments; $\hat{U}_{n}^{(1)}, \hat{U}_{n}^{(2)}$, and $\hat{U}_{n}^{(N-1)}$ are the polarisation transformation matrices of the PCs in the middle sections. The lengths of the nonlinear media and middle sections are denoted by $L_{i}$ and $L_{0}$, respectively. The emission spectrum of the biphotons generated from $\mathbf{b}$ a $20 \mathrm{~cm}$ PPSF, and $\mathbf{c}$ a cascade structure consisting of three identical $20 \mathrm{~cm}-\mathrm{long}$ PPSFs connected with two $6 \mathrm{~m}$-long SMF28 ${ }^{\text {TM}}$; The subset shows discretization of the frequency modes
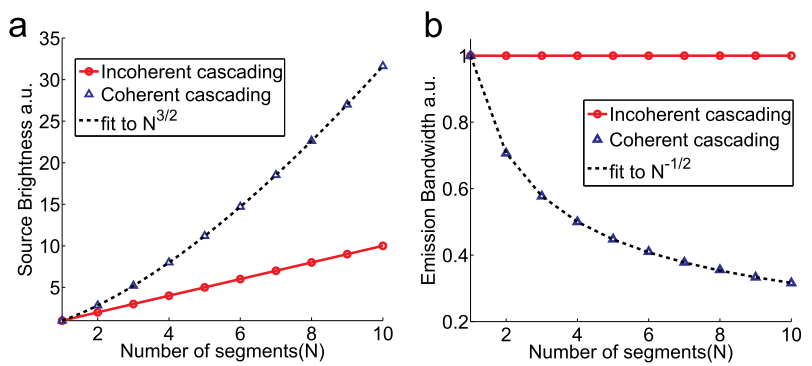

Fig. 3 Trade-off between the brightness and the emission bandwidth of the biphotons generated from the cascade structure with coherent and incoherent pumping of $N$ identical PPSFs. a Brightness scales linearly with respect to $N$ (or $L_{\mathrm{NL}}$ ) for incoherent cascading, while it scales by factor of $N^{3 / 2}$ (or $L_{N L}^{3 / 2}$ ) for coherent cascading. b The emission bandwidth is independent of $N$ (or $L_{\mathrm{NL}}$ ) for incoherent cascading, while it decreases with a factor of $N^{-1 / 2}$ (or $L_{\mathrm{NL}}^{-1 / 2}$ ) for coherent cascading

collective effects of the pump coherence, dispersion of the linear and nonlinear media, and the polarisation transformation applied in the middle section.

We model the unitary transformation of the middle section for signal and idler by a general unitary matrix of the form

$$
\hat{U}_{A(B)}\left(\theta, \phi_{1}, \phi_{2}\right)=\left(\begin{array}{cc}
e^{\mathrm{i} \phi_{1}} \cos \theta & -e^{\mathrm{i} \phi_{2}} \sin \theta \\
e^{-\mathrm{i} \phi_{2}} \sin \theta & e^{-\mathrm{i} \phi_{1}} \cos \theta
\end{array}\right)
$$

where $\theta$ is the angle of the polarisation rotation; note that $\phi_{1}$ and $\phi_{2}$ are the phase parameters, which define an arbitrary polarisation transformation; note that, $\phi_{1}$ and $\phi_{2}$ physically correspond to the birefringence introduced by the optical elements in the middle section, such as the PC. The collective transformation matrix of the middle section then becomes $\hat{R}_{n}=e^{i k_{0 n} L_{0}} \hat{U}_{n}\left(\theta, \phi_{1}, \phi_{2}\right)$. Given the polarisation transformation, we can use Eq. (5) and form the density matrix $\hat{\rho}=\left\langle\mathrm{B}_{\text {tot }}\right\rangle_{\text {avg }}^{-1}\left|\psi_{\text {CAS }}\right\rangle\left\langle\psi_{\text {CAS }}\right|$ (so that $\operatorname{Tr}(\hat{\rho})=1$ ) to characterise the polarisation state of the biphotons generated from the cascade structure.

Degree of polarisation entanglement. In order to study the effect of cascading on the degree of polarisation entanglement, we limit ourselves to transformation of the form $\hat{U}_{n}\left(\theta=\phi_{1}=\phi_{2}=0\right)$. The ensemble-averaged density matrix of the biphoton state in polarisation bases $\left(|\mathrm{HH}\rangle_{\omega_{A}, \omega_{B}},|\mathrm{HV}\rangle_{\omega_{A}, \omega_{B}},|\mathrm{VH}\rangle_{\omega_{A}, \omega_{B}},|\mathrm{VV}\rangle_{\omega_{A}, \omega_{B}}\right)$ can be written as:

$$
\begin{aligned}
& \left\langle\hat{\rho}_{\theta=\phi_{1}=\phi_{2}=0}^{\text {type ll }}\right\rangle_{\text {avg }}=\left\langle\mathrm{B}_{\text {tot }}\right\rangle_{\text {avg }}^{-1} \int \mathrm{d} \omega_{P} \mathrm{~d} \omega_{A} \mathrm{~d} \omega_{B}\left|\mathcal{A}_{0}^{\text {type ll }} \operatorname{af}\left(\omega_{P}\right) L_{1} \operatorname{sinc}\left(\frac{\Delta k_{\text {viv }}^{(1)} L_{1}}{2}\right)\right|^{2} \delta \\
& \times\left\{1+\left|U_{4 P}\right|^{2}+2\left|U_{4 P}\right| e^{\frac{-\left|\Delta T_{0}+\Delta \tau_{1}\right|}{\tau_{C}}} \cos \left(\Delta k^{(0)} L_{0}+\Delta k_{\mathrm{VHV}}^{(1)} L_{1}+\Phi_{P}\right)\right\} \\
& \times\left(\begin{array}{cccc}
0 & 0 & 0 & 0 \\
0 & 1 & e^{-\mathrm{i} 2 \Lambda} & 0 \\
0 & e^{\mathrm{i} 2 \Lambda} & 1 & 0 \\
0 & 0 & 0 & 0
\end{array}\right) .
\end{aligned}
$$

When $\int \mathrm{d} \omega_{A} \mathrm{~d} \omega_{B} \mathrm{e}^{\mathrm{i} 2 \Lambda}=0$, the density matrix for both pump coherence conditions has zero concurrence. ${ }^{54}$ This is due to the walk-off between the two biphoton polarisation states $|\mathrm{HV}\rangle_{\omega_{A}, \omega_{B}}$ and $|\mathrm{VH}\rangle_{\omega_{A}, \omega_{A}}$, which is introduced by the nonlinear segments. The walk-off in the cascade structure can usually be compensated by placing a birefringent element in the path of biphotons. However, it is more desirable to use nonlinear media with $\Lambda \ll 1$ (such as poled-fibres ${ }^{51}$ ), especially when dealing with complex configurations ${ }^{55}$ consisting of multiple cascaded nonlinear segments. The use of such nonlinear media $(\Lambda \ll 1)$ in the cascade structure also allows us to, for example, preserve polarisation entanglement (if present) and simultaneously perform spectral shaping, similar to what we mentioned in previous sections.

Shaping the polarisation state of the biphotons. Now we study the role of polarisation transformation in shaping the polarisation state of the biphotons generated from the cascade structure. We consider a polarisation rotation of the form $\hat{U}_{n}\left(\theta=\frac{\pi}{4}, \phi_{1}=\phi_{2}=0\right)$, for 

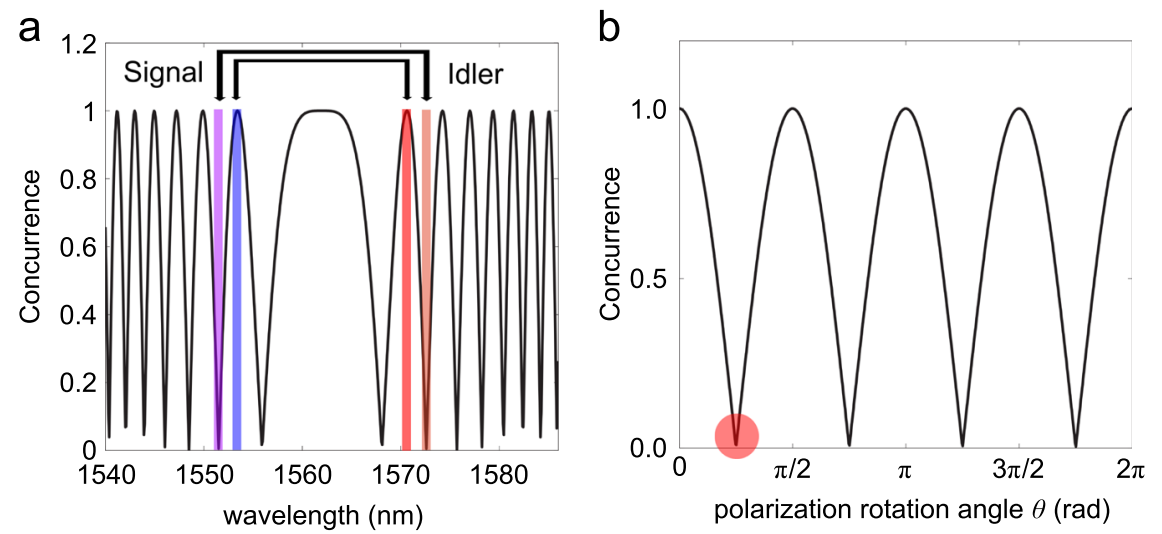

Fig. 4 a Concurrence as a function of signal and idler wavelengths for coherent cascading of two 30-cm-long PPSFs connected via a 3-m-long SMF $28^{\mathrm{TM}}$ (used as the middle section). The polarisation transformation is set to $\hat{U}_{n}\left(\theta=\frac{\pi}{4}, \phi_{1}=\phi_{2}=0\right)$. For certain signal (idler) wavelengths, such as those denoted by blue (red) strips closer to the degeneracy point, the concurrence is 1 , while for the adjacent strips, the concurrence is $\mathbf{0}$. $\mathbf{b}$ Concurrence as a function of the angle of the polarisation rotation in the middle section $(\theta)$ for incoherent cascading; the designated red circle corresponds to $\hat{U}_{n}\left(\theta=\frac{\pi}{4}, \phi_{1}=\phi_{2}=0\right)$

which the density matrix of the biphoton state becomes:

$$
\begin{aligned}
\left\langle\hat{\rho}_{\theta=\frac{\pi}{4}, \phi_{1}=\phi_{2}=0}^{\text {type }}\right\rangle_{\text {avg }}=\left\langle\mathrm{B}_{\text {tot }}\right\rangle_{\text {avg }}^{-1} \int \mathrm{d} \omega_{P} \mathrm{~d} \omega_{A} \mathrm{~d} \omega_{B}\left|\mathcal{A}_{0}^{\text {type II }} \operatorname{af}\left(\omega_{P}\right) L_{1} \operatorname{sinc}\left(\frac{\Delta k_{\mathrm{vHV}}^{(1)} L_{1}}{2}\right)\right|^{2} \delta \\
\times\left(\begin{array}{ccccc}
1 & -\rho_{1}^{*} & -\rho_{1}^{*} & -1 \\
-\rho_{1} & 1 & 1 & \rho_{2} \\
-\rho_{1} & 1 & 1 & \rho_{2} \\
-1 & \rho_{2}^{*} & \rho_{2}^{*} & 1
\end{array}\right),
\end{aligned}
$$

where

$$
\begin{aligned}
& \rho_{1}=\left|U_{4 P}\right| e^{\frac{-\left|\Delta \tau_{0}+\Delta \tau_{1}\right|}{\tau_{C}}} e^{\mathrm{i}\left(\Delta k^{(0)} L_{0}+\Delta k_{\mathrm{VHV}}^{(1)} L_{1}+\Phi_{P}-\Gamma_{B}\right),} \\
& \rho_{2}=\left|U_{4 P}\right| e^{\frac{-\left|\Delta \tau_{0}+\Delta \tau_{1}\right|}{\tau_{C}}} e^{\mathrm{i}\left(\Delta k^{(0)} L_{0}+\Delta k_{\mathrm{VHV}}^{(1)} L_{1}+\Phi_{P}-\Gamma_{A}\right) .}
\end{aligned}
$$

Note that we have assumed the nonlinear segments satisfy $\Lambda \ll 1$. For coherent cascading $\left(\left|\Delta \tau_{0}+\Delta \tau_{1}\right| \ll \tau_{C}\right)$, the matrix in Eq. (13) is wavelength-dependent due to $\rho_{1}$ and $\rho_{2}$ elements [see Eq. (14)]. In fact, it can be shown that for a small wavelength range of the signal and idler photons, the concurrence varies between 0 and 1 (see Fig. 4a). Note that the signal and idler photons are still spectrally correlated, while the degree of polarisation entanglement varies with respect to signal and idler wavelengths. The effect shown in Fig. 4a is a direct consequence of simultaneously manipulating the dispersion and birefringence of the middle section.

For incoherent cascading $\left(\left|\Delta \tau_{1}\right| \leq \tau_{C} \ll\left|\Delta \tau_{0}\right|\right)$, on the other hand, $\rho_{1}, \rho_{2} \rightarrow 0$ in Eq. (13) and the density matrix has now zero concurrence for the entire signal and idler wavelength range. Here the variation of the concurrence is a result of polarisation rotation, and mixing of the two maximally polarisation-entangled biphoton states. In fact, it can be shown that by varying $\theta$ in $\hat{U}_{n}\left(\theta, \phi_{1}=\phi_{2}=0\right)$, we can vary the value of the concurrence between 0 and 1, obtaining a biphoton state with arbitrary degree of polarisation entanglement (see Fig. 4b). Note that here we only considered type-II SPDC for both pump coherence conditions; however, in practice, two biphoton sources with differing SPDC phase-matchings can be combined within our cascade structure to generate an arbitrary biphoton polarisation state.

\section{Experiment}

A cw tunable 780-nm external-cavity diode-laser (ECDL, Toptica DL PRO) with a coherence time of $\tau_{C} \approx 3 \mu$ s (coherence length of $L_{C} \approx$ $1 \mathrm{~km}$ ) is used as a pump for coherent cascading. For incoherent cascading, we either decrease the time-averaged pump coherence by modifying the external cavity or separately pump the two PPSFs while the biphotons still travel in a common path (see Methods section). The pump power is adjusted for a pair generation rate of approximately $10^{6}$ pairs $\mathrm{s}^{-1}$, for which the probability of multi-pair generation is so small that it can be ignored. To demonstrate biphoton shaping in the spectral and polarisation domains, we add $5 \mathrm{~m}$ of SMF $28^{\text {TM }}$ alongside an inline polarisation controller (PC2 in the inset of Fig. 5) to manipulate the dispersion and birefringence of the middle section.

For our proof-of-principle demonstration, three types of measurements are performed on each individual PPSF sample, as well as the cascade structure as a whole (Fig. 5): (1) Measurement of the biphoton spectrum to observe the spectral interference, and to obtain the emission bandwidth of the biphotons; (2) a coincidence measurement to quantify the biphoton brightness; and (3) quantum state tomography ${ }^{56}$ (QST) to study the polarisation state of the biphotons under various polarisation transformations. The detection apparatus consists of two single photon detectors (SPDs, IDQ ID220), and a time-interval analyzer (TIA, Hydraharp 400). The biphoton spectrum is measured with an in-house fibre spectrometer ${ }^{57}$ (Fig. 5b). The spectral resolution of our spectrometer is approximately $0.75 \mathrm{~nm}$ $(100 \mathrm{GHz})$, limited primarily by the time jitter of our detectors.

For our setup, we choose two similar PPSFs by using the approach mentioned in the Methods section. The type- 0 and type-II SPDC emission spectra of the two PPSFs are shown in Fig. 6. We observe the spectral overlap $(\sim 80 \mathrm{~nm})$ is large around the degeneracy point, which allows us to obtain interference [predicted in Eq. (8)] in the spectrum of the biphotons generated from the cascade structure.

Biphoton spectral properties: coherent cascading

We cascade the two PPSFs and perform type-0 and type-II SPDC, depending on the pump wavelength used (Fig. 7). The emission spectra of the biphotons at the output of the cascade structure are shown in Fig. 7a, b. Note that we have not yet applied any polarisation rotation in the middle section $\left[\hat{U}_{n}\left(\theta=0, \phi_{1}, \phi_{2}\right)\right.$, see Eq. (11)]. The spectral interference fringes appear in the biphoton spectrum due to the dispersion of a 5-m-long SMF $28^{\mathrm{TM}}$ in the middle section, which connects the two fibrepigtailed PPSFs. The less-than-unity fringe visibility is mainly due to the spectral and brightness discrepancy between the two PPSFs. In our case, the fringe visibility of type-0 SPDC $(\sim 86 \%)$ is almost similar to what is observed for type-II SPDC ( 81\%) 


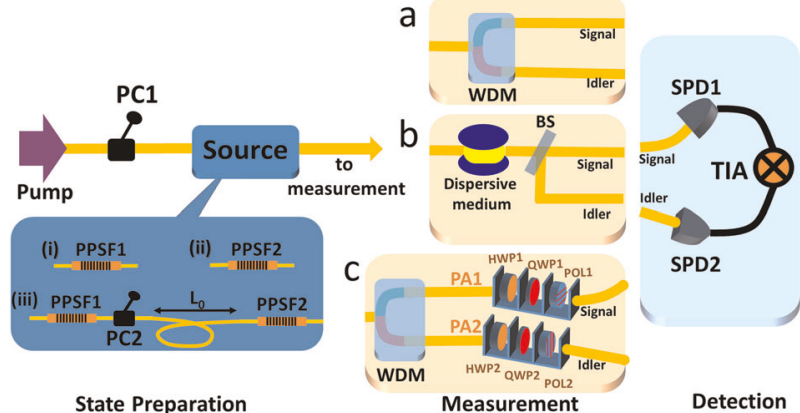

Fig. 5 Experimental setup. The source under test, illustrated in the inset, is pumped by a tunable $\mathrm{cw}$ diode laser. a A standard L-C band wavelength-division multiplexer (WDM) is used to separate signal (shorter wavelength) and idler (longer wavelength) photons into two different fibres for coincidence measurement. b For spectral measurements, a dispersive medium (20 km Corning SMF28 $\left.{ }^{\mathrm{TM}}\right)$, and a beam splitter (BS) are used as a fibre spectrometer that extracts the biphoton spectrum. The overall detection time jitter is $200 \mathrm{ps}$, based on which we select our coincidence time window to be $256 \mathrm{ps}$ for spectral measurements. The nominal dispersion-length product of the $20 \mathrm{~km}$ fibre spool is $340 \mathrm{ps} \mathrm{nm}^{-1}$, which gives a spectral resolution of $0.75 \mathrm{~nm}$ in our measurement. c For the QST experiment, two sets of HP 8169 A polarisation analyzers (PAs) are used, each of which includes a quarter waveplate (QWP), a half waveplate (HWP), and a polarizer (POL)
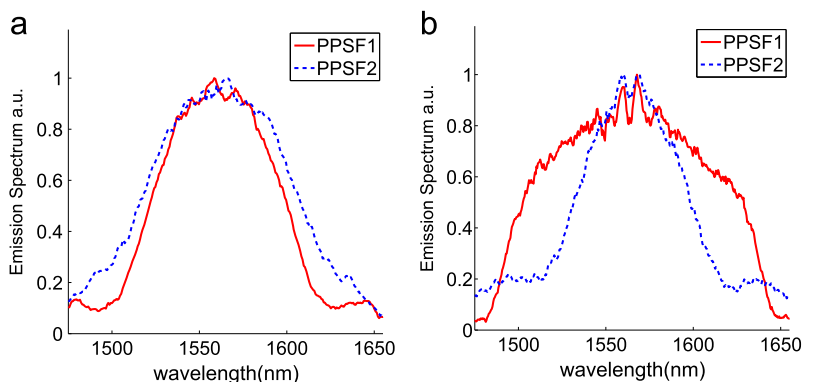

Fig. 6 Emission spectrum of each PPSF for $\mathbf{a}$ type-0 and $\mathbf{b}$ type-II SPDC; the emission spectra of the two segments are similar and overlap over a large bandwidth of $\sim 80 \mathrm{~nm}$

around the degeneracy wavelength, where the emission spectra of the two PPSFs are well matched (see Fig. 6). However, as we go further from the degeneracy wavelength, the discrepancy in the spectra of the two PPSFs increases (especially for type-II), and the fringe visibility of type- 0 and type-II drops to $\sim 80 \%$ and $\sim 50 \%$, respectively.

To highlight the effect of the pump coherence on the biphoton interference, we now decrease the time-averaged coherence of the pump (see Methods) and measure the spectrum of the biphotons at the output of the cascade structure for the type- 0 SPDC process. We use type- 0 since the emission spectra of the two PPSFs are similar, so that the initial assumption of identical emission spectra of the two nonlinear sources holds true. The fringe visibility disappears in Fig. 7a (red trace), and the biphoton spectrum is now just an incoherent sum of the two individual PPSF spectra; this is in a good agreement with our simulation result shown in Fig. 7c.

Biphoton spectral properties: incoherent cascading

In this section, we study the brightness and emission spectrum of the biphotons generated from the cascade structure under incoherent pumping. To quantify the biphoton brightness $\left[\left\langle B_{\text {tot }}\right\rangle_{\text {avg }}\right.$ in Eq. (10)], we measure the equivalent quantity, the coincidence rate of the biphotons (see Supplement, Section 8), for each individual sample as well as the cascade structure. We chose type-0 SPDC (pumped at $780.05 \mathrm{~nm}$ ) since the emission spectra of the two PPSFs largely overlap (see Fig. 6a), allowing us to observe the variation in the emission bandwidth of the biphotons.

We first pump each PPSF and measure the coincidence rates with respect to the pump power. Taking into account the effect of loss for the pump and the signal (idler) fields, the expected coincidence rate for the cascade structure becomes:

$R_{\text {exp }}=\eta_{A(B), 2}^{2} R_{\mathrm{PPSF} 1}+\eta_{P, 1} R_{\mathrm{PPSF} 2}$,

where $\eta_{\mathrm{A}(\mathrm{B}), 2}$ is the transmission of the signal (idler) field from the output of the PPSF1 to the output of the PPSF2, and $\eta_{P, 1}$ is the transmission of the pump field from the input of PPSF 1 to the input of PPSF2; $R_{\mathrm{PPSF} 1}$ and $R_{\mathrm{PPSF} 2}$ are the coincidence rate of the first and the second PPSF, respectively. We then use separate pumping technique (see Methods) to ensure incoherent cascading and measure the coincidence rate of the biphotons generated from the cascade structure and compare it with $R_{\exp }$ in Eq. (15). Note that the polarisation transformation in the middle section is set to $\hat{U}_{n}\left(\theta=0, \phi_{1}, \phi_{2}\right)$ [see Eq. (11)] during our measurements. The result in Fig. 8 a shows that for incoherent cascading, the brightness increases additively, and therefore scales linearly with the total nonlinear interaction length.

We then measure the emission spectrum of the biphotons generated from the cascade structure. As can be seen in Fig. 8b, the emission spectrum is the arithmetic mean of the two individual PPSF's spectra due to almost equal contribution of the two PPSFs at the output of the cascade structure. The result in Fig. $8 \mathrm{~b}$ also shows no bandwidth reduction, which indicates that the emission bandwidth in incoherent cascading becomes independent of total nonlinear interaction length inside the cascade structure.

Biphoton polarisation state: incoherent cascading

We now study the degree of polarisation entanglement and the polarisation state of the biphotons generated from the cascade structure by considering two specific transformations: (1) $\hat{U}_{n}\left(\theta=0, \phi_{1}, \phi_{2}\right)$, and (2) $\hat{U}_{n}\left(\theta=\frac{\pi}{4}, \phi_{1}, \phi_{2}\right)$. We first characterize the polarisation state of the biphotons generated from each PPSF when pumped for type-II SPDC at $782.05 \mathrm{~nm}$. Results in Fig. 9a, b show that both PPSFs generate biphoton states with a high concurrence, and high fidelity to triplet state $\left|\psi^{+}\right\rangle$(see also Table 1).

Degree of polarisation entanglement. The setup for cascading is similar to that of Fig. 5, except that we separately pump the two PPSFs (see Methods) to ensure incoherent cascading. This method of pumping helps us to precisely control the pairwise contributions of each PPSF segment in the final quantum state and at the same time enables us to shape the polarisation state of the biphotons. We now change the settings of PC2 (see Fig. 5) so that there would be no polarisation rotation in the middle section [ $\hat{U}_{n}\left(\theta=0, \phi_{1}, \phi_{2}\right)$, see Eq. (11)] and then measure the biphoton state again. It can be seen from Fig. $9 c$ that the measured density matrix corresponds to a highly polarisation-entangled state. Note that due to the negligible value of $\Lambda$ for the PPSFs, no walk-off is introduced between $|\mathrm{HV}\rangle_{\omega_{A}, \omega_{B}}$ and $|\mathrm{VH}\rangle_{\omega_{A}, \omega_{B}}$, and the degree of the polarisation entanglement remains unchanged after cascading.

Shaping the polarisation state of the biphotons. By applying a polarisation rotation of $\theta=\frac{\pi}{4}$ in the middle section $\left[\hat{U}_{n}\left(\theta=\phi_{1}=\frac{\pi}{4}, \phi_{2}=0\right)\right]$, the density matrix of the output state changes into the one shown in Fig. 9d. The concurrence drops to $\sim 0.1$ despite both PPSF segments individually generating highconcurrence polarisation-entangled biphotons. This value of the concurrence is consistent with the one predicted in Fig. $4 \mathrm{~b}$ and 

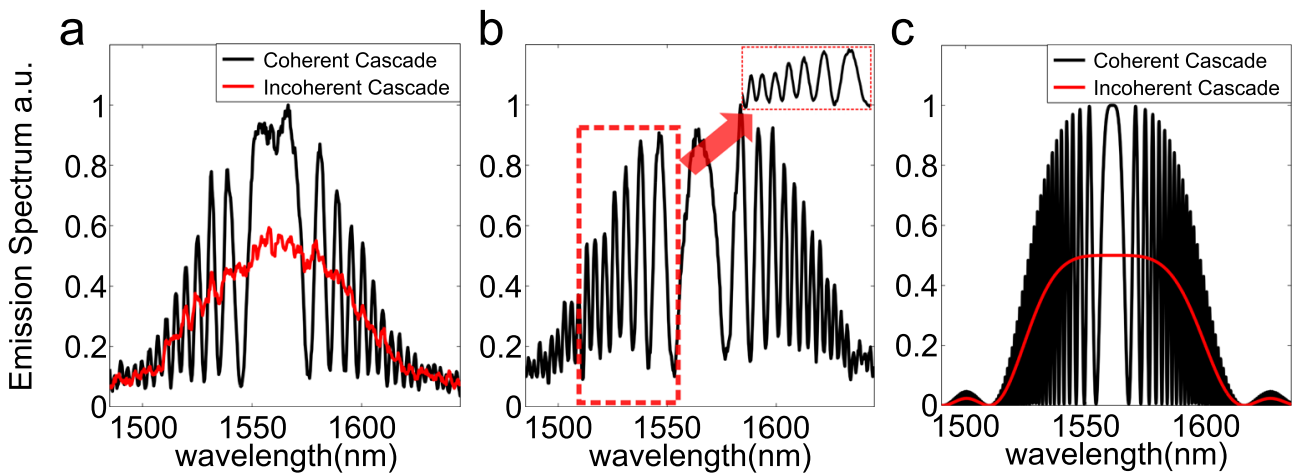

Fig. 7 a The emission spectrum of the biphotons generated from the cascade structure for type-0 SPDC (pumped at $780.05 \mathrm{~nm}$ ) when $\mid \Delta \tau_{1}+$ $\Delta \tau_{0} \mid \ll \tau_{C}$ (coherent cascade), and $\left|\Delta \tau_{1}\right| \leq \tau_{C} \ll\left|\Delta \tau_{0}\right|$ (incoherent cascade, red trace). $\mathbf{b}$ The emission spectrum of the biphotons generated from the cascade structure for type-II SPDC, when coherently pumped at $782.05 \mathrm{~nm}$; the inset shows the effect of the dispersion in the middle section on discretization of the frequency components. c Simulation result of the biphoton spectrum for type- 0 SPDC cascade; the cascade structure consists of two identical PPSFs, each of length $25 \mathrm{~cm}\left(=L_{1}=L_{2}\right)$, connected by an SMF $28^{\mathrm{TM}}$ patchcord of length $L_{0}=5 \mathrm{~m}$
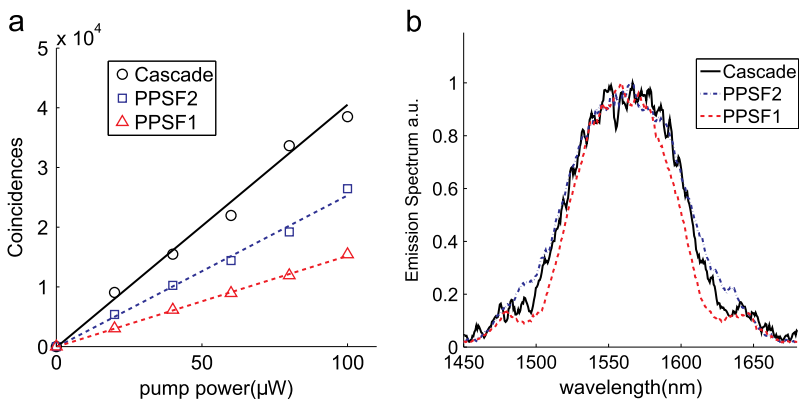

Fig. 8 a Coincidence rates are plotted as a function of pump power for type-0 SPDC. Symbols are measured data, while the solid and dashed lines are linear fits to the data points. For PPSF1, the displayed data points are the measured coincidence rates corrected by $\eta_{\mathrm{A}(\mathrm{B}), 2}^{2}$. For PPSF2, the displayed data points are the measured coincidence rates corrected by $\eta_{\mathrm{P}, 1}$. The error bars are so small that they cannot be shown in the figure. b Type-0 SPDC spectra of the individual segment, and the cascaded. Due to nearly equal contribution of each PPSF sample in the output, the biphoton spectrum at the output of the cascade structure is the average of the two PPSF spectra, and shows no bandwidth reduction

suggests that, for $0 \leq \theta \leq \frac{\pi}{4}$, we can arbitrary tune the concurrence between 0 and 1 . Note that here we have only considered type-II SPDC with two polarisation transformations; however, by applying various transformations $\hat{U}_{n}\left(\theta, \phi_{1}, \phi_{2}\right)$ with PC2 in the middle section and utilizing different SPDC phase-matchings, one can generate any biphoton polarisation states.

\section{DISCUSSION}

We have shown here that cascading biphoton sources in a common-path configuration can be used as a versatile tool to simultaneously tailor the frequency and polarisation DOFs of entangled photons. In this strategy, the pump coherence plays a major role in obtaining various biphoton states. With a longcoherence pump, the entire cascade structure can be considered as one unified source, capable of generating biphotons with tunable spectral properties ${ }^{58,59}$; in fact, one can obtain various biphoton spectra (Fig. 7) simply by engineering the dispersion of the linear medium in the middle section. For example, by cascading counter-propagating path-entangled biphoton sources, ${ }^{60}$ one could obtain biphoton frequency combs (similar to the works reported earlier ${ }^{1,22}$ ) of constant spacing whose free spectral range can be tuned by manipulating the dispersion of the middle section; note that, this can be done without any dispersion modification of the nonlinear medium.

Since our spectral and polarisation shaping techniques share the same configuration, we can simultaneously control biphotons in both DOFs. For example, by coherently pumping the cascade structure and manipulating the dispersion and birefringence property of the middle section, we can generate biphotons whose degree of polarisation entanglement is frequency-dependent (Fig. 4a); this new effect, arising from the interplay between coherence and entanglement, directly links the entanglement existing in the polarisation DOF to the frequency DOF of biphotons.

With incoherent pumping, the effects arising from biphotonbiphoton amplitude interference disappear, and the final state will become an incoherent mixture of the individual states generated from each nonlinear segment (see Figs. 8 and 9). The immediate application would be the ability to increase the brightness of the biphoton sources (at the expense of greater noise) by increasing the total nonlinear interaction length without sacrificing the emission bandwidth of the generated biphotons (see Figs. 3 and 8). In addition, the incoherent cascade scheme allows us to generate arbitrary biphoton polarisation states, ${ }^{13,14}$ and also control the degree of polarisation entanglement of biphotons (see Figs. $4 \mathrm{~b}$ and 9d). We remark that our configuration greatly simplifies the schemes previously used for generating arbitrary biphoton polarisation state ${ }^{13,14}$ and removes the requirement for phase stabilization and pump coherence due to its common-path configuration.

It is worth mentioning that using linear and nonlinear materials with negligible group birefringence (such as poled-fibres ${ }^{51}$ ) and small dispersion is of great importance in the cascade strategy as no walk-off between different biphoton polarisation states is essentially introduced. This feature allows us to preserve polarisation entanglement (if present), or generate arbitrary polarisation states ${ }^{13,14}$ without the need for complex walk-off compensation schemes. This can also be beneficial for the configurations recently proposed for generating multi-photon entanglement, ${ }^{55}$ which often involve complex scheme of multiple cascaded nonlinear media. In addition, nonlinear media with small dispersion generate broadband biphotons and with the simple spectral shaping technique presented here, they can serve as versatile quantum sources for various quantum information processing applications. ${ }^{1,2}$

The technique presented in this work can also be generalised to all other waveguide-based photon-pair sources, including those in integrated photonics devices, and one can use the effect of biphoton-biphoton amplitude interference to tune the properties of entangled photons, not only in the frequency and polarisation DOFs, but also in other DOFs such as path and orbital angular momentum as well. From this perspective, the cascade strategy 
a

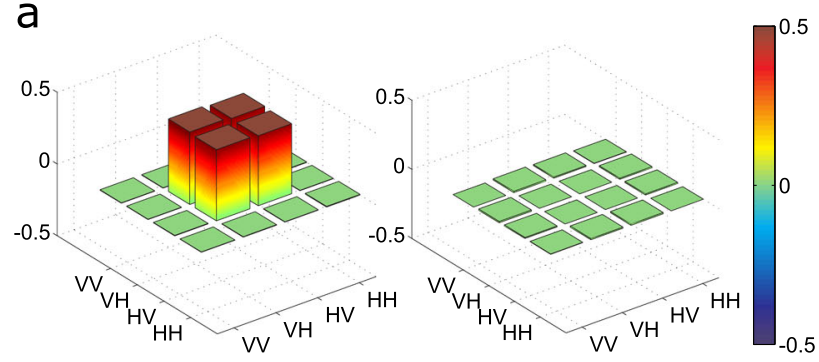

b

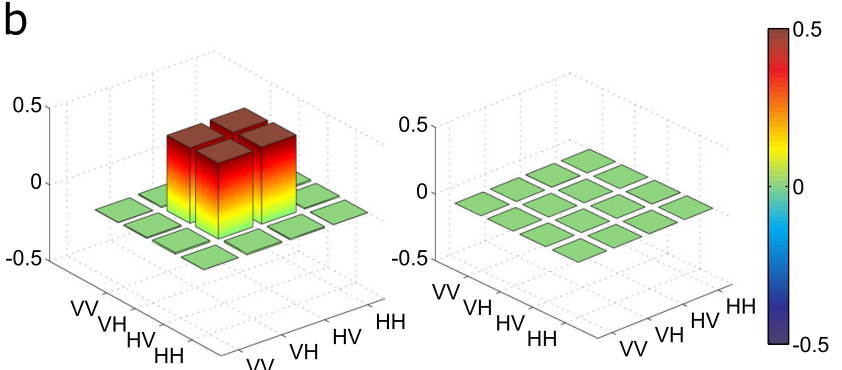

C

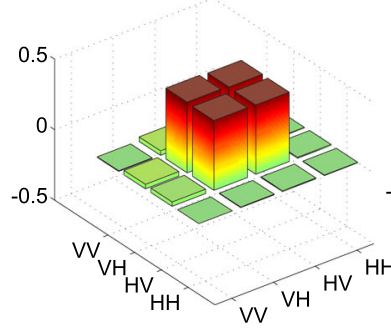

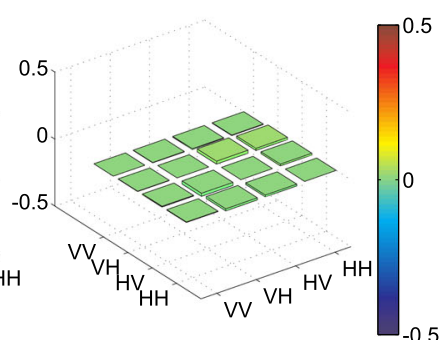

d

d -0.5
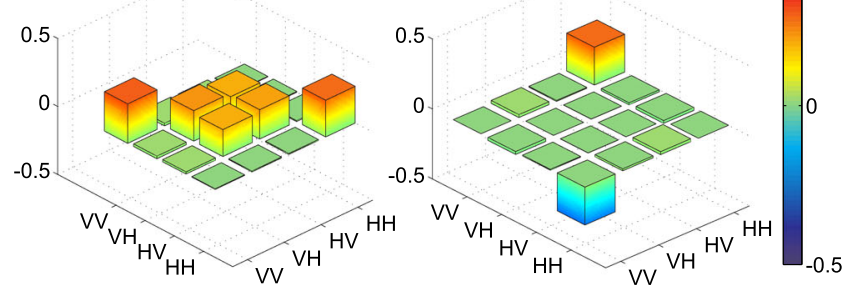

Fig. 9 Real and imaginary part of the output density matrix of a PPSF1, b PPSF2 and cascade structure corresponding to the polarisation transformation (c), $\hat{U}_{n}\left(\theta=\phi_{1}=\phi_{2}=0\right)(\mathbf{d})$, and $\hat{U}_{n}\left(\theta=\phi_{1}=\frac{\pi}{4}, \phi_{2}=0\right)$ in the middle section. Note that the relative contributions of the two samples at the output are set to be similar (48\% from PPSF1 and 52\% from PPSF2) by adjusting the pump power for each one of them

can be invaluable for generating a host of entangled-photon states that could be useful for quantum information processing, quantum sensing, and the study of the foundations of quantum mechanics.

\section{METHODS}

Choosing PPSF samples

The SHG spectrum of several PPSF samples are examined with a cW tunable laser (Agilent $8164 \mathrm{~A}$ ) in the $1550-1565 \mathrm{~nm}$ wavelength range. ${ }^{61}$ Depending on the input polarization of the fundamental lightwave, the type-0 or type-Il phase-matching can be observed. ${ }^{61}$ Two PPSFs whose SHG peaks and SPDC spectra are well-matched are then selected as nonlinear segments for our cascade structure.

\section{Fibre spectrometer}

The biphotons generated in the cascade structure are sent to a dispersive medium ( $20 \mathrm{~km}$ of Corning SMF-28), which maps their wavelengths onto the arrival time at the single photon detectors. ${ }^{57}$ After time-tagged detection with single photon detectors, the spectrum of the biphotons can be recovered by translating the time delays into wavelength. ${ }^{46,57}$ The minimum resolution of our spectrometer depends primarily on the timing jitter of the single photon detectors. ${ }^{46}$ As the overall detection time-jitter is $\sim 200 \mathrm{ps}$, we chose our coincidence time window to be $256 \mathrm{ps}$ for these measurements. Based on this coincidence window and the nominal dispersion-length product of the $20 \mathrm{~km}$ fibre spool, which is $340 \mathrm{ps} \mathrm{nm}{ }^{-1}$, we can obtain spectral resolution of $\sim 0.75 \mathrm{~nm}$ with our spectrometer.

\section{Incoherent pumping schemes}

Depending upon suitability, one of two following methods is used to achieve incoherent pumping: (1) We decrease the effective coherence time of the pump laser by periodically modulating the cavity length of ECDL (the $780 \mathrm{~nm}$ laser) so that its time-averaged linewidth (as measured by a Fabry-Perot spectrometer) increases, effectively reducing the pump temporal coherence; (2) We pump the two PPSFs separately while the biphotons still travel in a common path. Since the two pump fields reaching the PPSF segments travel in two different and unstabilized fibre paths, no coherence is preserved between the two fields, guaranteeing incoherent cascading.
Table 1. Fidelity, concurrence, and purity $\left[\operatorname{Tr}\left(\hat{\rho}^{2}\right)\right]$ of the biphoton state measured in polarisation basis

\begin{tabular}{lccc}
\hline Output states & Fidelity to $\left|\psi^{+}\right\rangle$ & Concurrence & Purity \\
\hline PPSF1 & $98.5 \pm 0.2 \%$ & $0.98 \pm 0.004$ & 0.988 \\
PPSF2 & $99.2 \pm 1.4 \%$ & $0.99 \pm 0.02$ & 0.985 \\
Cascading under & $99.2 \pm 1.8 \%$ & $0.985 \pm 0.003$ & 0.986 \\
$\hat{U}_{n}\left(\theta=\phi_{1}=\phi_{2}=0\right)$ & & & \\
Cascading under & $55 \pm 0.7 \%$ & $0.1 \pm 0.01$ & 0.482 \\
$\hat{U}_{n}\left(\theta=\phi_{1}=\frac{\pi}{4}, \phi_{2}=0\right)$ & & & \\
\hline
\end{tabular}

\section{DATA AVAILABILITY}

Data are available from the authors upon reasonable request.

\section{ACKNOWLEDGEMENTS}

The authors would like to thanks the anonymous reviewer for the comments regarding the pump coherence. This work is supported by Natural Sciences and Engineering Research Council of Canada (NSERC), grant no. (RGPIN-2014-06425, RGPIN-2014-05, RGPAS 462021-2014). Natural Sciences and Engineering Research Council of Canada (RGPIN-2014-06425, RGPIN-2014-05, RGPAS 462021-2014).

\section{AUTHOR CONTRIBUTIONS}

A.R. and L.Q. devised the experiment. J.E.S. and A.R. developed the theoretical framework for the cascade structure. A.R. performed the experiment, with help from E.Y.Z and C.C. Fabrication of the nonlinear devices was performed by A.V.G. and P.G.K. All authors contributed to the writing of the manuscript.

\section{ADDITIONAL INFORMATION}

Supplementary information accompanies the paper on the npj Quantum Information website (https://doi.org/10.1038/s41534-019-0188-1).

Competing interests: The authors declare no competing interests.

Publisher's note: Springer Nature remains neutral with regard to jurisdictional claims in published maps and institutional affiliations. 


\section{REFERENCES}

1. Kues, M. et al. On-chip generation of high-dimensional entangled quantum states and their coherent control. Nature 546, 622-626 (2017).

2. Reimer, C. et al. High-dimensional one-way quantum processing implemented on d-level cluster states. Nat. Phys. 15, 148-153 (2018).

3. Walther, P. et al. Experimental one-way quantum computation. Nature 434, 169-176 (2005).

4. Jennewein, T., Simon, C., Weihs, G., Weinfurter, H. \& Zeilinger, A. Quantum cryptography with entangled photons. Phys. Rev. Lett. 84, 4729 (1999).

5. Barreiro, J. T., Langford, N. K., Peters, N. A. \& Kwiat, P. G. Generation of hyperentangled photon pairs. Phys. Rev. Lett. 95, 260501 (2005).

6. Hardy, L. Nonlocality for two particles without inequalities for almost all entangled states. Phys. Rev. Lett. 71, 1665 (1993).

7. Shalm, L. K. et al. Strong loophole-free test of local realism. Phys. Rev. Lett. 115, 250402 (2015)

8. Qian, X.-F., Vamivakas, A. N. \& Eberly, J. H. Entanglement limits duality and vice versa. Optica 5, 942-947 (2018).

9. Lukens, J. M. \& Lougovski, P. Frequency-encoded photonic qubits for scalable quantum information processing. Optica 4, 8-16 (2017).

10. Lu, H.-H. et al. Quantum interference and correlation control of frequency-bin qubits. Optica 5, 1455-1460 (2018).

11. Lu, H.-H. et al. A controlled-NOT gate for frequency-bin qubits. npj Quantum Inf. 5, 24 (2019).

12. Zhong, T. et al. Photon-efficient quantum key distribution using time-energy entanglement with high-dimensional encoding. New. J. Phys. 17, 022002 (2016).

13. White, A. G., James, D. F. V., Eberhard, P. H. \& Kwiat, P. G. Nonmaximally entangled states: production, characterization, and utilization. Phys. Rev. Lett. 83, 3103 (1999).

14. Peters, N. A. et al. Maximally entangled mixed states: creation and concentration. Phys. Rev. Lett. 92, 133601 (2004).

15. Wei, T.-C. et al. Synthesizing arbitrary two-photon polarization mixed states. Phys. Rev. A 71, 032329 (2005).

16. Cinelli, C., Di Nepi, G., De Martini, F., Barbieri, M. \& Mataloni, P. Parametric source of two-photon states with a tunable degree of entanglement and mixing: experimental preparation of Werner states and maximally entangled mixed states. Phys. Rev. A 70, 022321 (2004).

17. Jaeger, G., Shimony, A. \& Vaidman, L. Two interferometric complementarities. Phys. Rev. A 51, 51-54 (1995).

18. Pe'er, A., Dayan, B., Friesem, A. A. \& Silberberg, Y. Temporal shaping of entangled photons. Phys. Rev. Lett. 94, 073601 (2005).

19. Lu, H.-H., Odele, O. D., Leaird, D. E. \& Weiner, A. M. Arbitrary shaping of biphoton correlations using near-field frequency-to-time maapping. Opt. Lett. 43, 743-746 (2018).

20. Lu, Y. J., Campbell, R. L. \& Ou, Z. Y. Mode-Locked two-photon states. Phys. Rev. Lett. 91, 163602 (2003).

21. Olislager, L. et al. Frequency-bin entangled photons. Phys. Rev. A 82, 013804 (2010).

22. Xie, Z. et al. Harnessing high-dimensional hyperentanglement through a biphoton frequency comb. Nat. Photonics 9, 536-542 (2015).

23. Nasr, M. B. et al. Ultrabroadband biphotons generated via chirped quasi-phasematched optical parametric down-conversion. Phys. Rev. Lett. 100, 183601 (2008).

24. Brańczyk, A. M., Fedrizzi, A., Stace, T. M., Ralph, T. C. \& White, A. G. Engineered optical nonlinearity for quantum light sources. Opt. Express 19, 55-65 (2011).

25. Bonfrate, G., Pruneri, V., Kazansky, P. G., Tapster, P. \& Rarity, J. G. Parametric fluorescence in periodically poled silica fibers. Appl. Phys. Lett. 75, 2356 (1999).

26. Zhu, E. Y. et al. Direct generation of polarization-entangled photon pairs in a poled fiber. Phys. Rev. Lett. 108, 213902-213905 (2012).

27. Chekhova, M. V. \& Ou, Z. Y. Nonlinear interferometers in quantum optics. Adv. Opt. Photonics 8, 108-155 (2016).

28. Yurke, B., McCall, S. L. \& Klaud, J. R. SU(2) and SU $(1,1)$ interferometers. Phys. Rev. A 33, 4033 (1986)

29. Kong, J. et al. Experimental investigation of the visibility dependence in a nonlinear interferometer using parametric amplifiers. Appl. Phys. Lett. 102, 011130 (2013).

30. Vered, R. Z., Shakhed, Y., Ben-Or, Y., Rosenbluh, M. \& Pe'er, A. Classical-to-quantum transition with broadband four-wave mixing. Phys. Rev. Lett. 114, 063902 (2015).

31. Shaked, Y., Pomerantz, R., Vered, R. Z. \& Pe'er, A. Observing the nonclassical nature of ultra-broadband bi-photons at ultrafast speed. New J. Phys. 16, 053012 (2014).

32. Jing, J., Liu, Z., Ou, Z. Y. \& Zhang, W. Realization of a nonlinear interferometer with parametric amplifiers. Appl. Phys. Lett. 99, 011110 (2011).

33. Lemieux, S. et al. Engineering the frequency spectrum of bright squeezed vacuum via group velocity dispersion in an SU $(1,1)$ interferometer. Phys. Rev. Lett. 117, 183601 (2016).

34. Manceau, M., Leuchs, G., Khalili, F. \& Chekhova, M. V. Detection loss tolerant supersensitive phase measurement with an SU $(1,1)$ interferometer. Phys. Rev. Lett. 119, 223604 (2017).
35. Klyshko, D. N. Ramsey interference in two-photon parametric scattering. JETP 77, 222 (1993).

36. Milonni, P. W., Fearn, H. \& Zeilinger, A. Theory of two-photon down-conversion in the presence of mirrors. Phys. Rev. A 53, 4556 (1995).

37. Burlakov, A. V. et al. Interference effects in spontaneous two-photon parametric scattering from two macroscopic regions. Phys. Rev. A 56, 3214-3225 (1997).

38. Zou, X. Y., Wang, L. J. \& Mandel, L. Induced coherence and indistinguishability in optical interference. Phys. Rev. Lett. 67, 318 (1991).

39. Ou, Z. Y., Wang, L. J., Zou, X. Y. \& Mandel, L. Evidence for phase memory in twophoton down-conversion through entanglement with the vacuum. Phys. Rev. A 41, 566 (1990).

40. Herzog, T. J., Rarity, J. G., Weinfurter, H. \& Zeilinger, A. Frustrated two-photon creation via interference. Phys. Rev. Lett. 72, 629 (1994).

41. Herzog, T. J., Kwiat, P. G., Weinfurter, H. \& Zeilinger, A. Complementarity and the quantum eraser. Phys. Rev. Lett. 75, 3034 (1995).

42. Wang, I. J., Zou, X. Y. \& Mandel, L. Induced coherence without induced emission. Phys. Rev. A 44, 4614 (1991).

43. Lemos, G. B. et al. Quantum imaging with undetected photons. Nature 512, 409-412 (2014).

44. Kulik, S. P. et al. Two-photon interference in the presence of absorption. JETP 98, 31-38 (2004).

45. Kalashnikov, D. A., Petrova, A., Kulik, S. \& Krivitsky, L. A. Infrared Spectroscopy with visible light. Nat. Photonics 10, 98-102 (2016).

46. Riazi, A. et al. Alignment-free dispersion measurement with interfering biphotons. Opt. Lett. 44, 1484-1487 (2019).

47. Liscidini, M., Helt, L. G. \& Sipe, J. E. Asymptotic fields for a Hamiltoninan treatment of nonlinear electromagnetic phenomena. Phys. Rev. A 85, 013833 (2012).

48. Helt, L. G., Liscidini, M. \& Sipe, J. E. How does it scale? Comparing quantum and classical nonlinear optical processes in integrated devices. J. Opt. Soc. Am. B 29, 2199-2212 (2012).

49. Loudon, R. The Quantum Theory of Light. (Oxford University Press, New York, 2000).

50. Hertel, I. V. \& Schulz, C. Atoms, Molecules and Optical Physics 2. (Springer, Berlin, 2015).

51. Chen, C. et al. Compensation-free broadband entangled photon pair sources. Opt. Express 25, 22667-22678 (2017).

52. Chen, C. et al. Turn-key diode-pumped all-fiber broadband polarizationentangled photon source. OSA Continuum 1, 981-987 (2018).

53. Yariv, A. \& Yeh, P. Photonics. (Oxford University Press, New York, 2007).

54. Wootters, W. K. Entanglement of formation of an arbitrary state of two qubits. Phys. Rev. Lett. 80, 2245-2248 (1998).

55. Krenn, M., Hochrainer, A., Lahiri, M. \& Zeilinger, A. Entanglement by path identity. Phys. Rev. Lett. 118, 080401 (2017).

56. James, D. F. V., Kwiat, P. G., Munro, W. J. \& White, A. G. Measurement of qubits. Phys. Rev. Lett. 64, 0523121-15 (2001).

57. Zhu, E. Y., Corbari, C., Kazansky, P. G. \& Qian, L. Self-calibrating fiber spectrometer for the measurement of broadband down-converted photon pairs. arXiv:1505.01226v1 (2015).

58. Riazi, A. et al. Quantum interferometry through cascading broadband entanglement sources, presented at Conference on Lasers and Electro-Optics (CLEO), 1-2 (San Jose, CA, 2018).

59. Su, J. et al. Versatile and precise quantum state engineering by using nonlinear interferometers. Opt. Express 27, 20479-20492 (2019).

60. Saravi, S., Pertsch, T. \& Setzpfandt, F. Generation of counterpropagating pathentangled photon pairs in a single periodic waveguide. Phys. Rev. Lett. 118, 183603 (2017).

61. Zhu, E. Y. et al. Measurement of $X^{(2)}$ symmetry in a poled fiber. Opt. Lett. 35, 1530-1532 (2010).

Open Access This article is licensed under a Creative Commons Attribution 4.0 International License, which permits use, sharing, adaptation, distribution and reproduction in any medium or format, as long as you give appropriate credit to the original author(s) and the source, provide a link to the Creative Commons license, and indicate if changes were made. The images or other third party material in this article are included in the article's Creative Commons license, unless indicated otherwise in a credit line to the material. If material is not included in the article's Creative Commons license and your intended use is not permitted by statutory regulation or exceeds the permitted use, you will need to obtain permission directly from the copyright holder. To view a copy of this license, visit http://creativecommons. org/licenses/by/4.0/.

(c) The Author(s) 2019 